\title{
A Survey on EMG Biofeedback System
}

\author{
Hemlata Shakya ${ }^{1}$ and Shiru Sharma ${ }^{2}$ \\ ${ }^{1}$ School of Biomedical Engineering \\ Indian Institute of Technology, (Banaras Hindu University) \\ Varanasi, India \\ ${ }^{2}$ School of Biomedical Engineering \\ Indian Institute of Technology, (Banaras Hindu University) \\ Varanasi, India
}

\begin{abstract}
In this paper describe a new technology of placing electrodes on user's muscle recording the electrical activity which is called as electromyography. EMG is an instrument that recording the electrical activity of the muscles. It is a diagnostic procedure to assess the health of muscles and the nerve cells that control them. In this paper reviews relating to the biofeedback used in physical rehabilitation. The physiological systems of the body which can be measured to provide biofeedback are the musculoskeletal system. EMG biofeedback useful in both musculoskeletal and neurological rehabilitation
\end{abstract}

Keywords: Electromyography, Biofeedback, Muscles

\section{Introduction}

EMG (Electromyography) is an instrument for measuring the electrical activity of the muscles. An EMG is a physiological parameter that measures the conducting function of nerves. When muscles are active, they produce an electrical current. The current is usually proportional to the level of the muscle activity. An EMG is also referred to as myogram.The EMG signal is a biomedical signal that measures electrical current generated in muscles during its contraction representing neuromuscular activities. The EMG signal recorded can be significantly affected by physiological parameters muscle, temperature, muscle cross section area and length. The EMG signal analysis is in clinical diagnosis and biomedical application.

EMG biofeedback is the process of measuring and transforming the physiological information from muscle into visual and audio signals. The EMG biofeedback training is to realize the risk of overloading working related to muscle pain [5].

EMG can be used to detect abnormal electrical activity of muscles. The EMG can also be used to detect weakness as opposed to weakness from reduced use because of pain or lack of motivation. It can also be used to isolate the level of nerve irritation or injury.

EMG uses surface electrode to detect a change in skeletal muscle activity, which is then fed back to the user usually by a auditory signal and visual signal. The EMG electrodes are surface electrode and needle electrodes are used. Surface electrodes which detect small voltage changes which arise from the working muscles. The electrode gel helps in converting the biological signals to electrical signals needed by the machine, and will also help to reduce some noise. Needle electrodes are widely used in clinical procedures in neuromuscular evaluations. The needle electrode is placed on the skin in to the muscle. The electrical activity is detected by this needle.

The EMG biofeedback can be used to increase activity in weak or paretic muscle or it can be used to facilitate a reduction in tone is a spastic one. It has been shown to be useful in both musculoskeletal and neurological rehabilitation. 


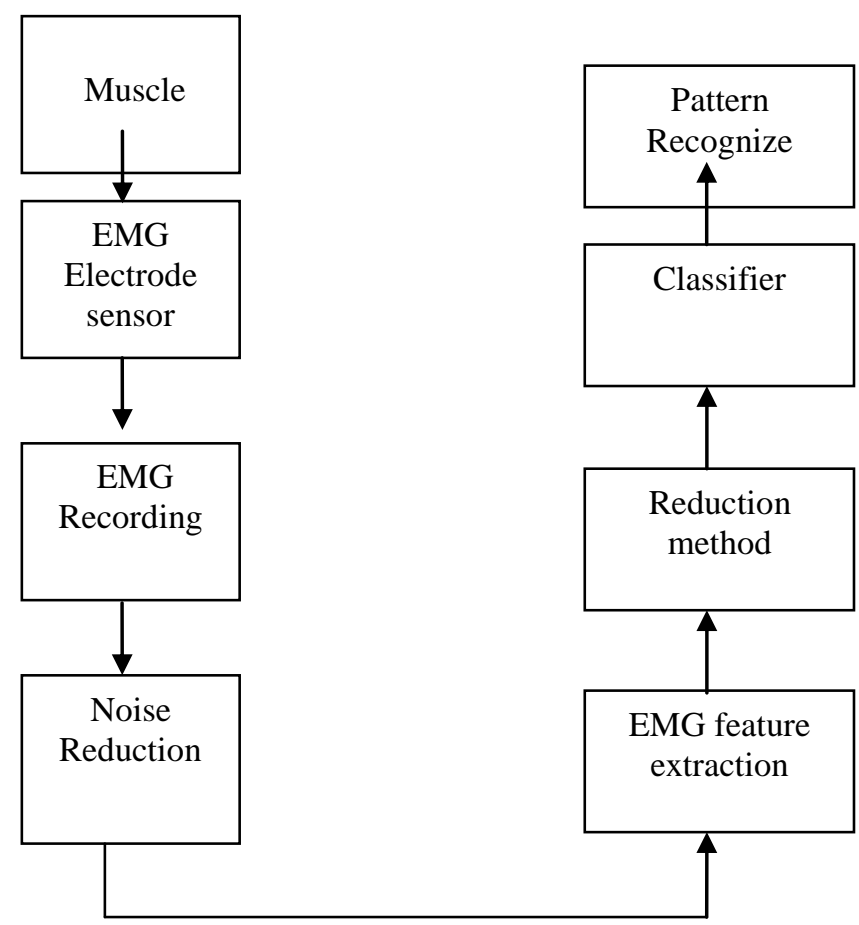

Figure 1. Block Diagram of EMG Biofeedback System

\section{Literature Survey}

Several research methods have been developed EMG biofeedback system so, discuss about papers:

In 1991, this paper describes an EMG biofeedback device and some preliminary data on the feasibility for clinical testing. It deals with the control of relaxation and contraction of skeletal muscle. The second generation EMG biofeedback device uses a constant current stimulus. Excitation of paralyzed muscle by electrical stimulation is an effective means of restoring controllable function.

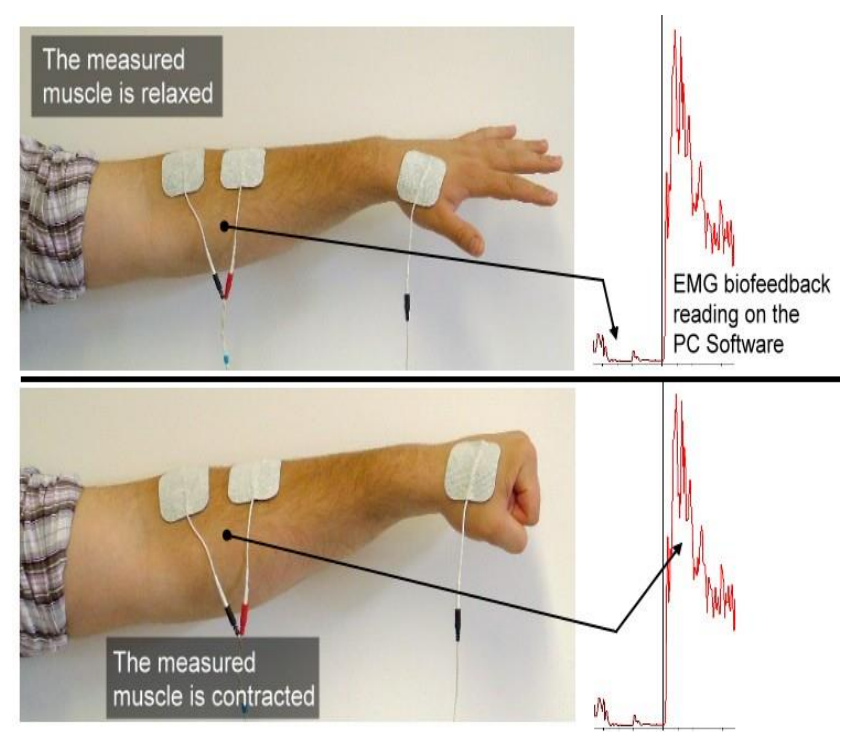

Figure 2. EMG Measured Muscle 
In 2003, this paper describe a computerized biofeedback system developed as part of a digital polygraph which is capable of acquire store, filter and measurement parameters online, retrieve data files from disk, analyze and process recorded data. The biofeedback system developed is easy to understand and use. Because it was based upon a versatile digital polygraph, it inherited in powerfulness to handle physiological data. The easy way one can use and set up different animation images, textual feedback and other feedback method is a good strength that can be helpful in the patient rehabilitation [3].

During the 2006, a real time multimodal biofeedback system for stroke patient rehabilitation. This paper developing experimental media systems that integrate task dependent physical therapy and cognitive stimuli within an interactive, multimodal environment. The use of biofeedback allows the patient who has sensor motor impairment to regain the ability to better discriminate a physiological response thereby better learning self control of that response. Our system situates participants in a multi-sensory engaging.

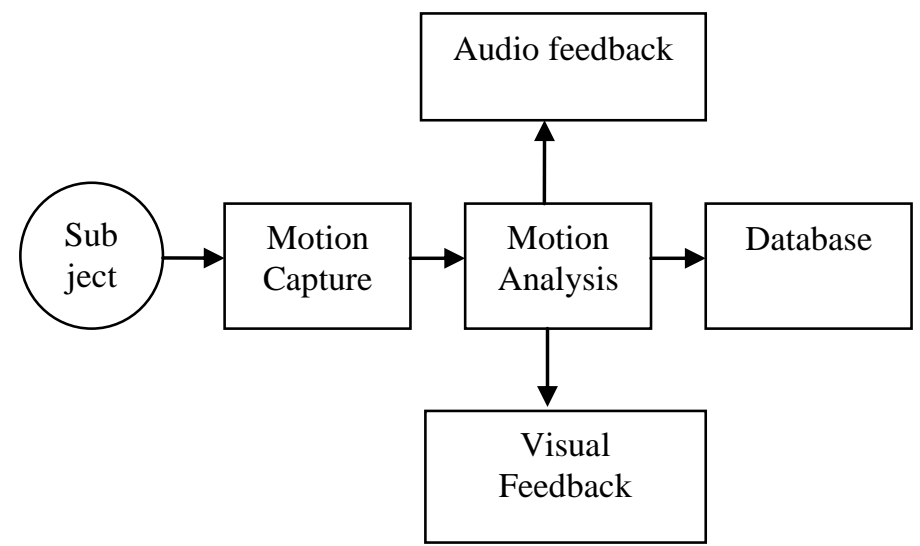

Figure 3. The Biofeedback System Diagram

In 2009, this paper presents an EMG biofeedback has been widely used for rehabilitation programs and in design orthotic and robotic device. These devices need calibration and recalibration which usually involves a set of maximum voluntarily contractions. The most used biofeedback is electromyogram of one of the muscle or the joint torque signal. This experiment on six healthy subjects and seven activation levels indicated that change in biofeedback type had a significant effect on the activation ratio of these two muscles [6].

The study in (2010), developing EMG is an important tool in a virtual reality based system for hand rotation and motion rehabilitation. The developed EMG biofeedback based virtual reality system enables the user to interact with virtual objects in real time with multiform feedback. VR which could provide a repetitive multimodal task oriented rehabilitation environment for patients to undertake self training in safety, is considered to be a suitable tool for medical health rehabilitation.

The combination of biofeedback and virtual offers more beneficial measurement which may help clinicians to improve the quality of treatment of motor disorder. The EMG biofeedback based VR system has been developed to provide an interactive training environment for hand motion rehabilitation. The developed balloon shooting training task includes hand rotation and grasping motions.

In 2010, the surface electromyogram is adopted as the bioelectrical signal and converted into an auditory signal. The sound conversion occurs inside a wearable device.

This paper introduces a wearable device for EMG auditory biofeedback device, activity using a light weight device.

In 2010, this paper describe a health relaxation system which can collect data from human body using sensors and electrode measure small changes in the electrical properties of the skin. 
The controls the movement with three major functions:

$>$ Filtering

$>$ Sensitivity

$>$ Ascent / Descent ratio

In 2012, this paper describes a real time rehabilitation training system with Electromyography feedback was developed based on AD sample card. The system for electromyography monitoring EMG signal and sEMG parameter which reflect muscle intensity and fatigue in time and frequency domain. The function of EMG signal parameters, analysation and calculation storage are also included. The result shows the system may provide some meaningful information about training processes that is direct to improve the training programs.

In 2013, this paper develops a differential EMG biofeedback training for children with attention deflect hyperactivity disorder. Differential EMG biofeedback training provides control method for neurofeedback training in ADHD research and effectively tested for feasibility on a child with ADHD.

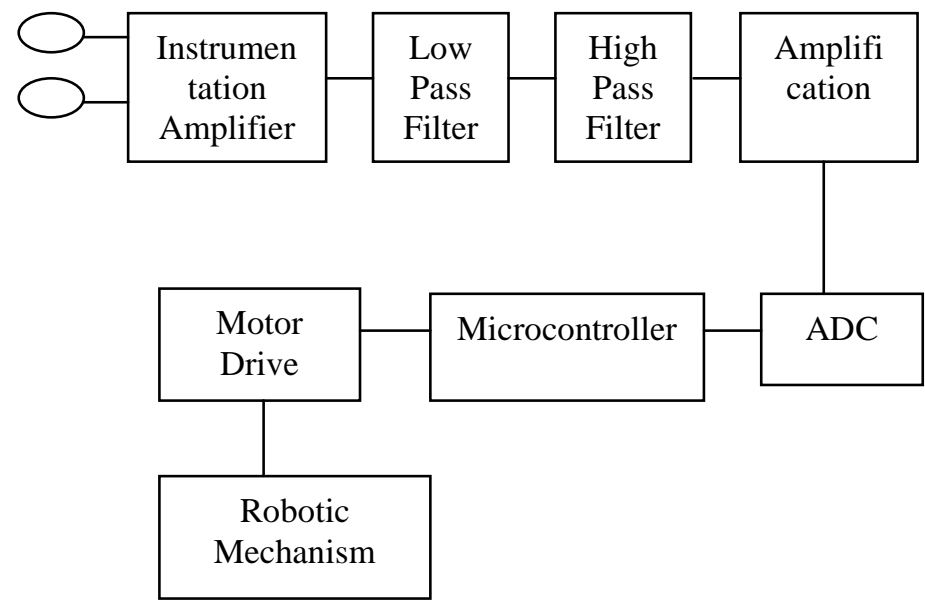

\section{Figure 4. Principle of EMG Biofeedback System}

In 2014, this paper presents implementation of the algorithm for the recognition of four movements fingers extension, flexion and redial and ulnar flexion. This paper introduces a new algorithm for real time classification of muscle activities. Design the system for neurorehabilitation of humans after brain injury.

\section{Conclusions}

This paper presents, biofeedback is a technique that uses monitoring instrument to measure and feedback information about muscle tension, skin temperature, heart rate. The biofeedback system developed is easy to understand and use. This paper has reviewed the EMG biofeedback applications that are currently being used in physical rehabilitation. This research in this field primary focuses on the use of biofeedback in rehabilitation of patient with disorders.

\section{References}

[1] F. Borgeat, B. Hade, L. M. Larouche and C. N. Bedwani, "Effect of Therapist's active presence on EMG biofeedback Training of Headache Patients", vol. 5, no. 2, (1980).

[2] J. A. Lucca and S. Jean Recchiuti, "Effect of Electromyograph Biofeedback on an Isometric Strengthening Program", vol. 63, February (1983), pp. 200-203. 
[3] L. C. Carvalho, H. F. Albuquerque, C. Pontes, M. T. Maia, D. P. R. Mangueira, L. V. Batista, "Computerized Biofeedback Tool: Application in EMG Biofeedback", International conference of the IEEE EMBS cancum, Mexico, (2003) September 17-21.

[4] Y. Tsubouchi and K. Suzuki, "Biotones: A Wearable Device for EMG Auditory Biofeedback", (2010) September 4.

[5] W. Sroykham Angngoen, W. Khemthong, S. Jalayondeja, W. Kajompredanon and Y. Thanangkul, "Effect of EMG Biofeedback on muscle activity in Computer work", International Conference on Biomedicl Engineering, (2012), pp. 1-4.

[6] F. Rahimi, J. P. Callaghan, F. Janabi-Sharifi and D. Wang, "EMG Biofeedback and Load Sharing Problem in assistive and Rehabilitation Orthotic Devices", International conference on the IEEE EMBS Minneapolis, Minisota, USA, (2009), September 2-6.

[7] S. Ma, M. Varley, L. Kwan Shark and J. Richards, "EMG Biofeedback based VR System for hand rotation and grasping rehabilitation", IEEE International Conference Information Visualization, (2010).

[8] S. Maurizio, M. D. Liechti, D. Brandeis, L. Jancke and R. Drechsler, "Differential EMG Biofeedback for Children with ADHD: A Control Method for Neurofeedback Training with a Case Illustration", appl Psychophysiol Biofeedback, (2013), pp. 109-119.

[9] O. M. Giggins, U. McCarthy Persson and B. Caulfield, "Biofeedback in rehabilitation", Journal of Neuroengineering and rehabilitation, (2013).

[10] E. Dursun, N. Dursun and D. Alican, "Effect of Biofeedback treatment on gait in children with cerebral palsy", Disability and rehabilitation, vol. 26, no. 2, (2004), pp. 116-120.

[11] A. J. Friddlund and J. T. Cacioppo, "Guidelines for Human Electromyographic Research", Psychophysiology, vol. 23, (1986), pp. 567-589.

[12] S. L. Wolf, "Electromyographic biofeedback application to stroke patients: a critical review", physical therapy, vol. 63, (1983), pp. 1448-1459.

[13] P. P. Roberto Merletti, "Electromyography physiology and noninvasive applications", Engineering I. press, Ed. John Wiley \& sons, Hoboken, New Jersey, (2004).

[14] D. Feng, P. Ji, W. Niu and A. Ji, "Quantitative Study on the Efficacy of Electromyograph Biofeedback Thearapy of Patients with Masticatory Spasm", IEEE, (2011)

[15] I. M. jovanov and D. B. Popovic, Member, IEEE, "EMG based Biofeedback with the Smarting System", Symposium on Neural Network Application in Electrical Engineering, (2014), pp. 25-27.

[16] N. Jirakittayakorn and Y. Wongsawat, "An EMG Instrument Designed for Bruxism Detection on Masseter Muscle", International Conference on Biomedical Engineering, (2014).

[17] M. Y. Lee, M. K. Wong, F. T. Tang, W. H. Chang and Y. L. Chen, "Cervical Traction Using EMG Biofeedback", IEEE Engineering in Medicine and Biology, (1996) June, pp. 83-87.

[18] S. Guangji, W. Li, M. A. Dengrong, F. Fan and N. Haijun, "The Design of a Rehabilitation Training System with EMG Feedback", International Conference on Biomedical Engineering and Biotechnology, IEEE, (2012).

[19] R. Strungaru, "EMG Biofeedback System”, Polytechnical Institute, IEEE, (1991), pp. 756-758.

[20] G. M. Lyons, P. Sharma, M. Baker, S. O'Malley and A. Shanahan, "A Computer Game Based EMG Biofeedback System for Muscle Rehabilitation", Engineering and Medicine and Biology Society, (2003), vol. 2, pp. 1625-1628.

[21] D. H. Rho, Y. J. Jeon, N. B. Lee and J. I. Chung, "Extraction of Parameters from EMG signals for the Biofeedback Electrical stimulation”, Engineering in Medicine and Biology, vol. 1, (2002), pp. 133-134. 
International Journal of Bio-Science and Bio-Technology

Vol.7, No.5 (2015) 